J. Clin. Chem. Clin. Biochem.

Vol. 24, 1986, pp. 233-242

(C) 1986 Walter de Gruyter \& Co.

Berlin - New York

\title{
Pediatric Reference Values for Calcium, Magnesium and Inorganic Phosphorus in Serum Obtained from Bhattacharya Plots for Data from Unselected Patients
}

\author{
By $W$. de Kieviet
}

Children's Hospital 'Emma Kinderziekenhuis' and Hospital 'Sint Lucas Ziekenhuis'

E. H. Slaats

Hospital 'Onze Lieve Vrouwe Gasthuis' and

N. G. G. M. Abeling

Children's Hospital 'Emma Kinderziekenhuis'

Amsterdam, The Netherlands

(Received January 30/November 29, 1985)

Summary: Pediatric reference values for total calcium, magnesium and inorganic phosphorus are given. The values were determined by using Bhattacharya plots for unselected data. The reference values for calcium (from 8320 values from patients) and magnesium (from 1231 values from patients) show only minor dependence on age. Reference values for ages up to 20 years for calcium were $2.14-2.64 \mathrm{mmol} / \mathrm{l}$ and for magnesium $0.57-1.12 \mathrm{mmol} / \mathrm{l}$. However, for inorganic phosphorus (3349 values from patients) a gradual decrease in concentration occurred throughout childhood from $1.56-2.29 \mathrm{mmol} / 1$ ( $<1$ year) to $1.03-1.78$ $\mathrm{mmol} / \mathrm{l}$ (> 15 years).

Pädiatrische Referenzwerte für Calcium, Magnesium und anorganisches Phosphat im Serum, ermittelt aus Bhattacharya-Diagrammen für Daten von unausgewählten Patienten

Zusammenfassung: Referenzwerte für Gesamt-Calcium, Magnesium und anorganisches Phosphat in der Kinderheilkunde werden mitgeteilt. Die Werte wurden mit Bhattacharya-Diagrammen für unausgewählte Daten ermittelt. Die Refereñzwerte für Calcium (aus 8320 Werten von Patienten) und Magnesium (aus 1231 Werten von Patienten) zeigen nur geringe Altersabhängigkeit. Die Referenzwerte für ein Lebensalter bis zu 20 Jahren betragen für Calcium 2,14- $, \dot{6}, \mathbf{m m o l} / 1$ und für Magnesium 0,57-1,12 mmol/1. Für anorganisches Phosphat (3349 Werte von Patienten) wurde jedoch ein allmählicher Abfall der Konzentration während der Kindheit von 1,56 bị $2,29 \mathrm{mmol} / 1$ ( $<1 \mathrm{Jahr}$ ) auf $1,03-1,78 \mathrm{mmol} / 1$ ( $>15 \mathrm{Jahre}$ ) beobachtet.

\section{Introduction}

A well known problem in pediatric clinical chemistry is the acquisition of adequate age-related reference values for routinely determined parameters. Detailed knowledge about the reference values is especially important with respect to those parameters that are closely related to growth and development. Therefore we chose serum calcium, magnesium and inorganic phosphorus for our study. 
In most statistical studies on reference values a "healthy" population is chosen $(1-3)$. Apart from the numerous difficulties of defining "healthy" and "normal", in practice it is nearly impossible to find a statistically acceptable number of "healthy" children, representative for the population of each particular hospital, yet large numbers are needed to cover the various age groups.

The use of routine laboratory data from any hospital has the great advantage of the easy availability of a large number of data from the hospital's own population; in the present case, this is our children's hospital.

A statistical method has been described using Bhattacharya plots for values from unselected patients for some haematological parameters (4). The method of Bhattacharya is based on the assumption that the reference range of results of a laboratory test is Gaussian shaped or can be transformed to it. The method offers the possibility of elimination of pathological data from the "normal" or reference population of data (5).

In this study the method was applied retrospectively, by using routine data collected over a 6 -year period to calculate pediatric reference values for serum calcium, magnesium and inorganic phosphorus.

\section{Materials and Methods}

Subjects studied

Data were derived from all the in- and outpatients of our children's hospital, whose serum had been routinely analysed for one or more of the three studied parameters between July 1977 and August 1983. The age distributions of the data for the three analytes are given in table 1 . Although both sexes were almost equally represented, the data were not processed separately.

One of the specialities of our children's hospital is pediatric oncology. A significant proportion of the test results is therefore derived from tumour patients receiving long term parenteral administration, which is monitored by regular determinations of electrolytes. This often gives multiple test results per patient for each of the parameters studied. In these cases only the first value of each series was taken into account.

Besides these patients, other relevant groups comprised children undergoing surgery, nephrological patients and children screened for problems of growth and development.

\section{Blood sampling and analytical methods}

For the three analytes studied, serum was used for analysis. The blood was obtained by venipuncture, as well as by capillary sampling, depending on the age of the patient and the number of tests requested. Most of the samples were obtained from fasting children, although many samples were collected in various non-fasting circumstances. The blood was collected, then allowed to clot for about $20 \mathrm{~min}$, after which the serum was separated from the cells by centrifugation at $3000 \mathrm{~min}^{-1}$ for 10 min. Analysis was performed on the day of specimen collection (calcium) or within three days (magnesium, inorganic phosphorus) after storage at $+4^{\circ} \mathrm{C}$.

All tests were performed with routine microchemical methods. Total calcium in serum was measured on a calcium analyser (Corning 940, Halstead, U. K.) by titration with ethyleneglycolbis(2-aminoethyl)-tetraacetate (EGTA) with calcein as indicator in an alkaline solution (6).

Magnesium in serum was measured colorimetrically by the xylidyl-blue method (Merckotest 3338, Merck, Darmstadt, FRG) (7).

Inorganic phosphorus in serum was measured after deproteinization with $100 \mathrm{~g} / \mathrm{l}$ trichloroacetic acid, by a phosphomolybdate method (8).

All reagents used were of analytical grade. The inter-assay coefficients of variation for control sera during the testing period were: for calcium, $<1.5 \%$; magnesium, $<5 \%$, and inorganic phosphorus, $<3 \%$.

\section{Statistical analysis}

The data were grouped according to age: age groups were created where necessary to obtain sufficient data per group. The number of tests per age or age group ranged from 224 (calcium; $17=20$ years old) to 1661 (calcium; $<1$ year old) (tab. 1) ${ }^{1}$ ). For each age or age group statistical calculations were performed according to Naus et al. (4). The reference intervals were calculated from $\left.\mu \pm 1.96 \sigma(95 \% \text { interval })^{2}\right)$.

\section{Results}

For all age groups (tab. 1) the raw data histograms for the three parameters showed Gaussian distribution patterns, with some pathological contamination superimposed on the extremities, mainly in the lower concentration part of the distributions. The Bhattacharya plot was linear for the main part of the data histograms. The calculated means $(\mu)$ and standard deviations $(\sigma)$ are given in table 1 .

The measured reference ranges (fig. 1) showed a minor, but statistically relevant age correlation for calcium and magnesium (Student's t-test: $\mathrm{p}<0.005$ ).

If the minor age dependence is neglected the data for all ages gave a reference range for calcium of $2.14-2.64 \mathrm{mmol} / 1$ (8329 data from patients) and for magnesium of $0.57-1.12 \mathrm{mmol} / 1$ (1231 data from patients).

The reference values for inorganic phosphorus showed a gradual decrease in concentration throughout childhood from $1.56-2.29 \mathrm{mmol} / 1$ (age $<1$ year) to $1.03-1.78 \mathrm{mmol} / \mathrm{l}$ (age > 15 years).

1) All distribution histograms are available upon request from the authors or from the editorial office of this journal.

2) A copy of the computer pogram in BASIC is available on request. 


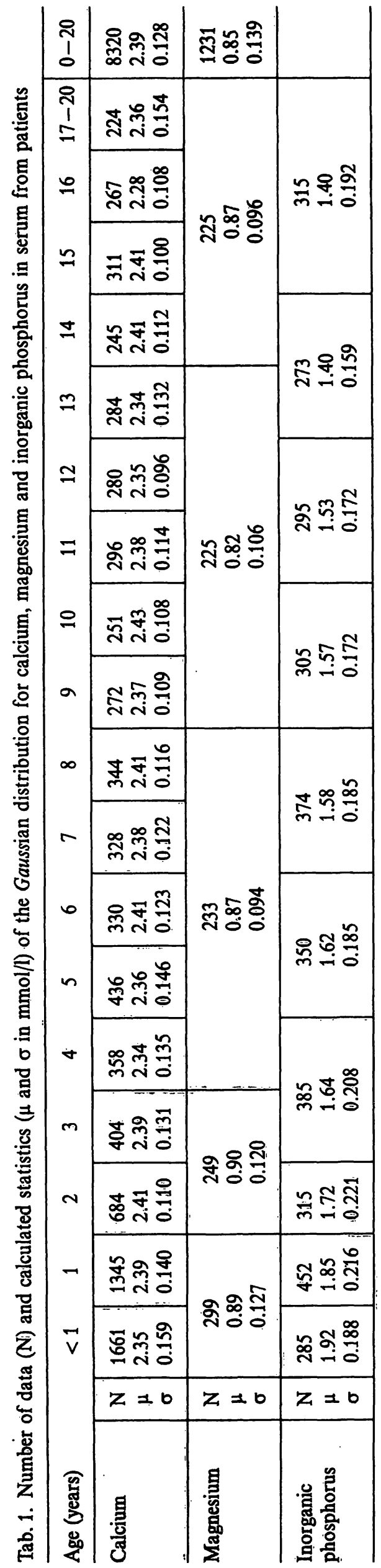

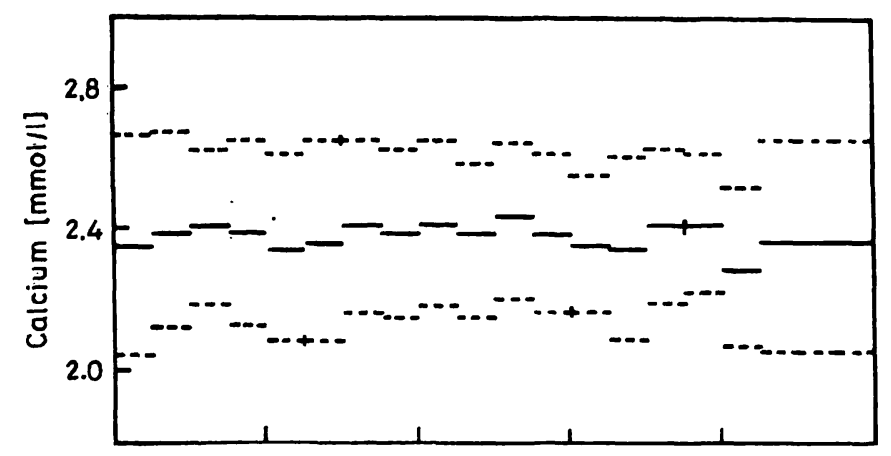
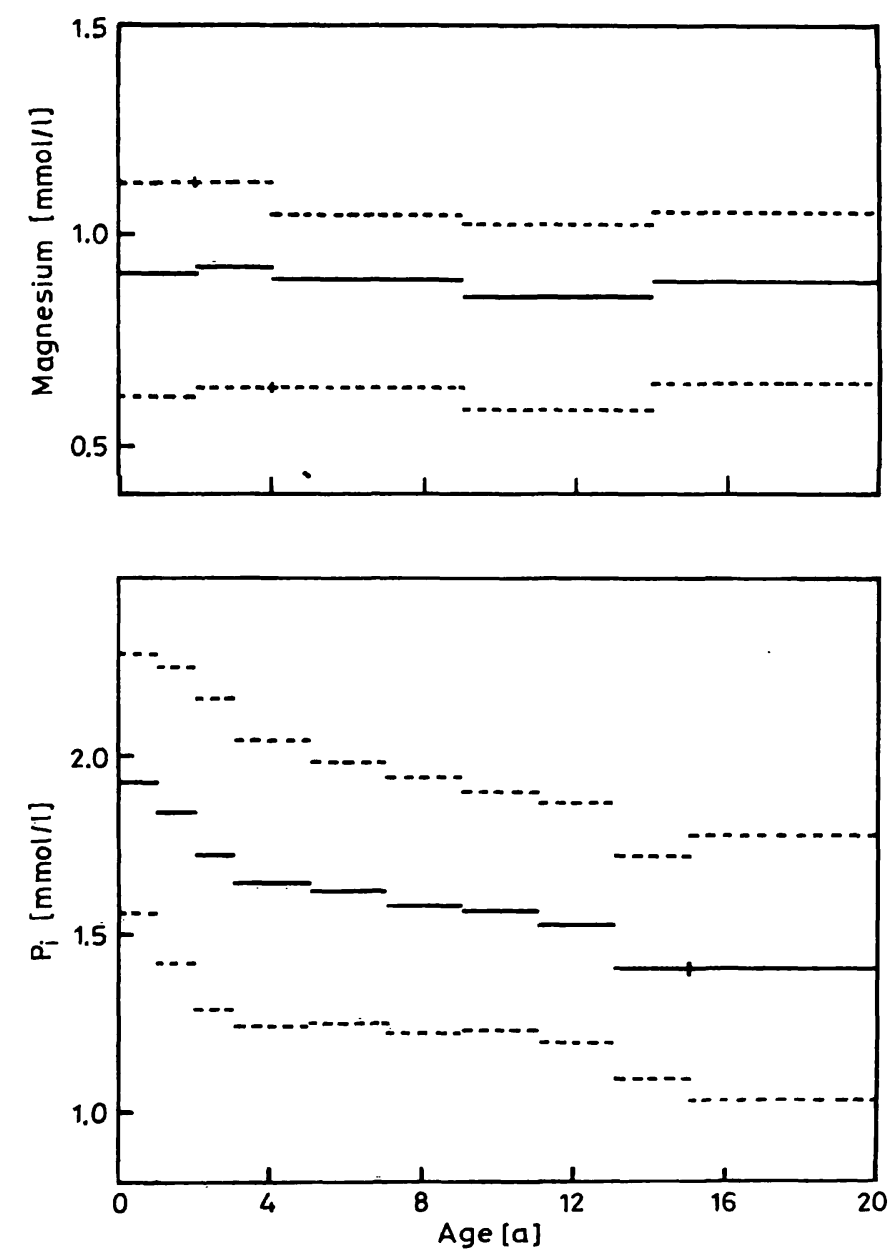

Fig. 1. Age dependence of the pediatric reference ranges of calcium, magnesium and inorganic phosphorus in serum ( $\mu \pm 1.96 \sigma ; 95 \%$ reference interval).

\section{Discussion}

Adequate reference values for the own hospital situation can be assessed by several methods. A representative group of the local population can be used and considered as healthy. Besides the problem of exclusion of pathology, this method is rather impractical in pediatrics, because blood has to be sampled from a reasonably sized group of healthy children per age. Moreover, the influence of hospitalization on the test results is neglected. 
Another approach is the use of the own hospital population. Reference values are assessed from the "healthy" part of the hospital population. Fewer discrepancies between the healthy and pathological subpopulations are then expected with regard to the composition of the population and the influence of hospitalization. The data can easily be obtained, even retrospectively. The disadvantage of these methods is the problem of adequate exclusion of pathological test results.

Several statistical methods based on unselected data from patients have been described $(5,9-15)$. Of these, a modified Bhattacharya approach has been found useful $(4,15)$. The modifications consist of a smoothing procedure on the raw-data histogram and a weighted linear-regression analysis for the construction of the Bhattacharya plot. Important conditions for the valid application of the Bhattacharya method are the Gaussian (or Gaussian-transformed $(15,16)$ data distributions of the "healthy-patient" data and the relatively small extent of contamination by pathological values. A selection of the patient data in "presumably healthy" and "presumably pathologic" in order to test the statistical procedure, introduces the problem of selection criteria as described above.
The reference range for calcium found in this study for our children's hospital population is in agreement with the ranges described previously for "healthy" volunteers $(1-3)$. It must be emphasized that newborns can have deviate calcium values (17). The reference range for calcium for $<1$ year cannot be taken for newborns.

The reference range for magnesium found for ages up to 20 years $(0.57=1.12 \mathrm{mmol} / \mathrm{l})$ is somewhat wider than given for "healthy children" $(0.70-0.95 \mathrm{mmol} / 1$ (1)), but the mean values are virtually the same. The adult range in the literature $(0.65-1.25 \mathrm{mmol} / \mathrm{l}$; dyechelation method) (1) fits well with the pediatric range now presented.

Previous reports of the decrease of inorganic phosphorus with age $(1,3)$ are reconfirmed in this study, using a greater number of data.

The conclusion can be drawn that the statistical method of Bhattacharya, with its limitations as described above, is useful in pediatrics for providing age-related reference values. The advantage is that reference values are derived from the same population that provides analytical material for the hospital laboratory. Large numbers of data can easily be collected to provide pediatric reference ranges for the own hospital situation.

\section{References}

1. Meites, S. (1981) Pediatric clinical chemistry, Second Ed., Am. Ass. for Clinical Chemistry Inc., Washington D. C.

2. Cheng, M. H., Lipsey, A. I., Blanco, V., Wong, H. T. \& Spiro, S. H. (1979) Clin. Chem. 25, 692-698.

3. Gómez, P., Coca, C., Vargas, C., Acebillo, J. \& Martinez, A. (1984) Clin. Chem. 30, 407-412.

4. Naus, A. J. M., Borst, A. \& Kuppens, P. S. (1980) J. Clin. Chem. Clin. Biochem. 18, 621 - 625 .

5. Bhattacharya, C. (1967) Biometrics 23, 115-135.

6. Schmid, R. W. \& Reiley, C. N. (1957) Anal. Chem. 29, 264-268.

7. Bohuon, C. (1962) Clin. Chim. Acta 7, 811-817.

8. Taussky, H. H. \& Shorr, E. (1953) J. Biol. Chem. 202, 675.

9. Becktel, J. (1970) Clin. Chim. Acta 28, 119-125.

10. Hoffmann, R. (1963) J. Am. Med. Ass. 185, 864-873.

11. Neumann, G. (1968) Clin. Chem. 14, 979-988.

12. Pryce, J. (1960) Lancet $I I, 333$.

13. Chy, S. Y., Cheung, P. \& Turkington, V. E. (1976) Clin. Biochem. 9, 198.

14. White, J. (1978) Clin. Chin. Acta 84, 353-360.

15. Naus, A. J. M. (1982) De berekeningen van referentiewaarden in de klinische chemie uit analysresultaten van een patiëntenpopulatie (in Dutch, summary in English). Thesis Maastricht, The Netherlands.

16. Boyd, J. C. \& Lacher, D. A. (1982) Clin. Chem. 28, 1735-1741.

17. Wilt, I., Trendelenburg, Chr. (1982) J. Clin. Chem. Clin. Biochem. 20, 235-242.

Dr. W. de Kieviet

Department of Clinical Chemistry

Children's Hospital

'Emma Kinderziekenhuis'

Spinozastraat 51

NL-1018 HJ Amsterdam 
Appendix: Distribution histograms

Calcium

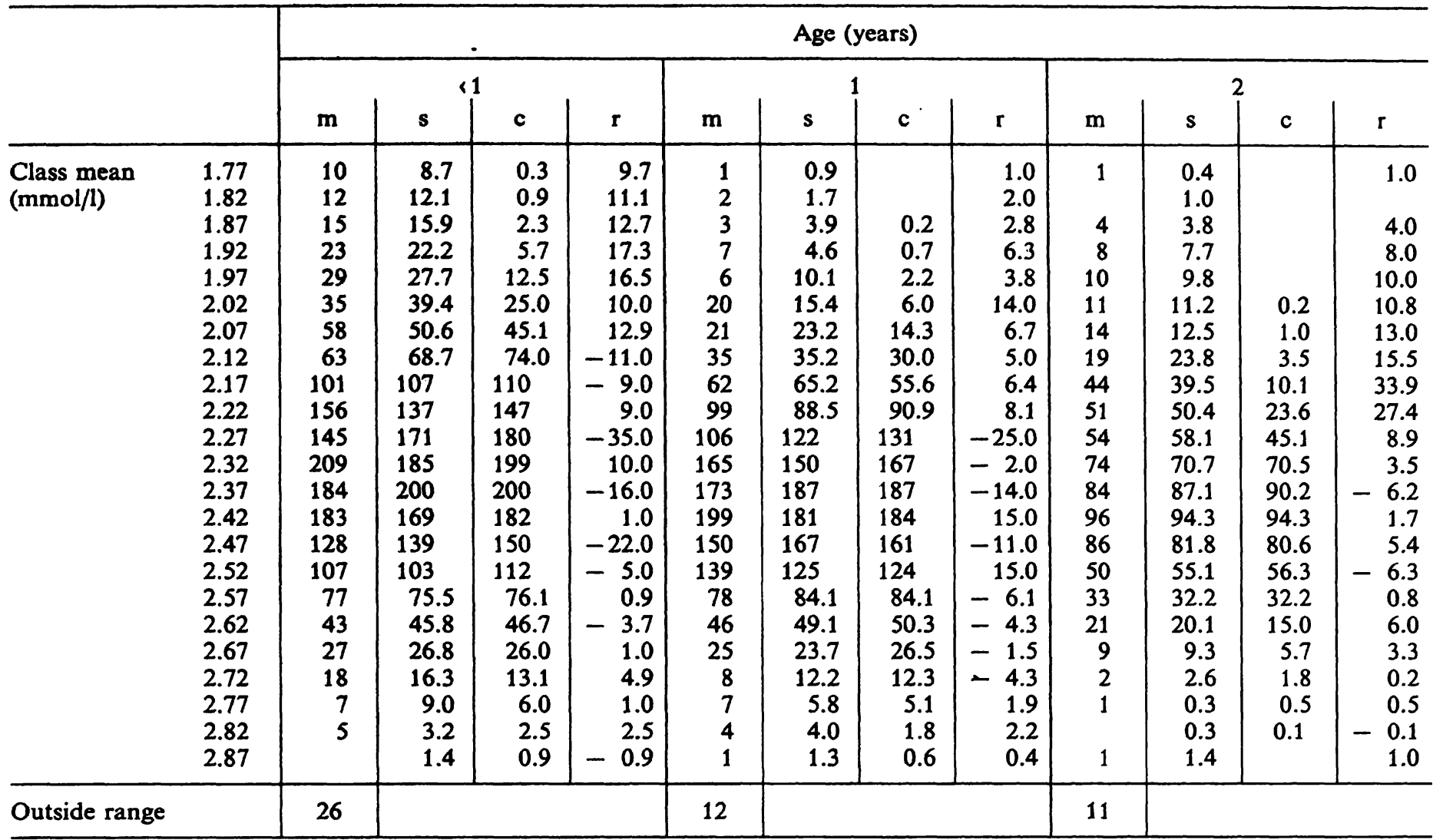

Calcium

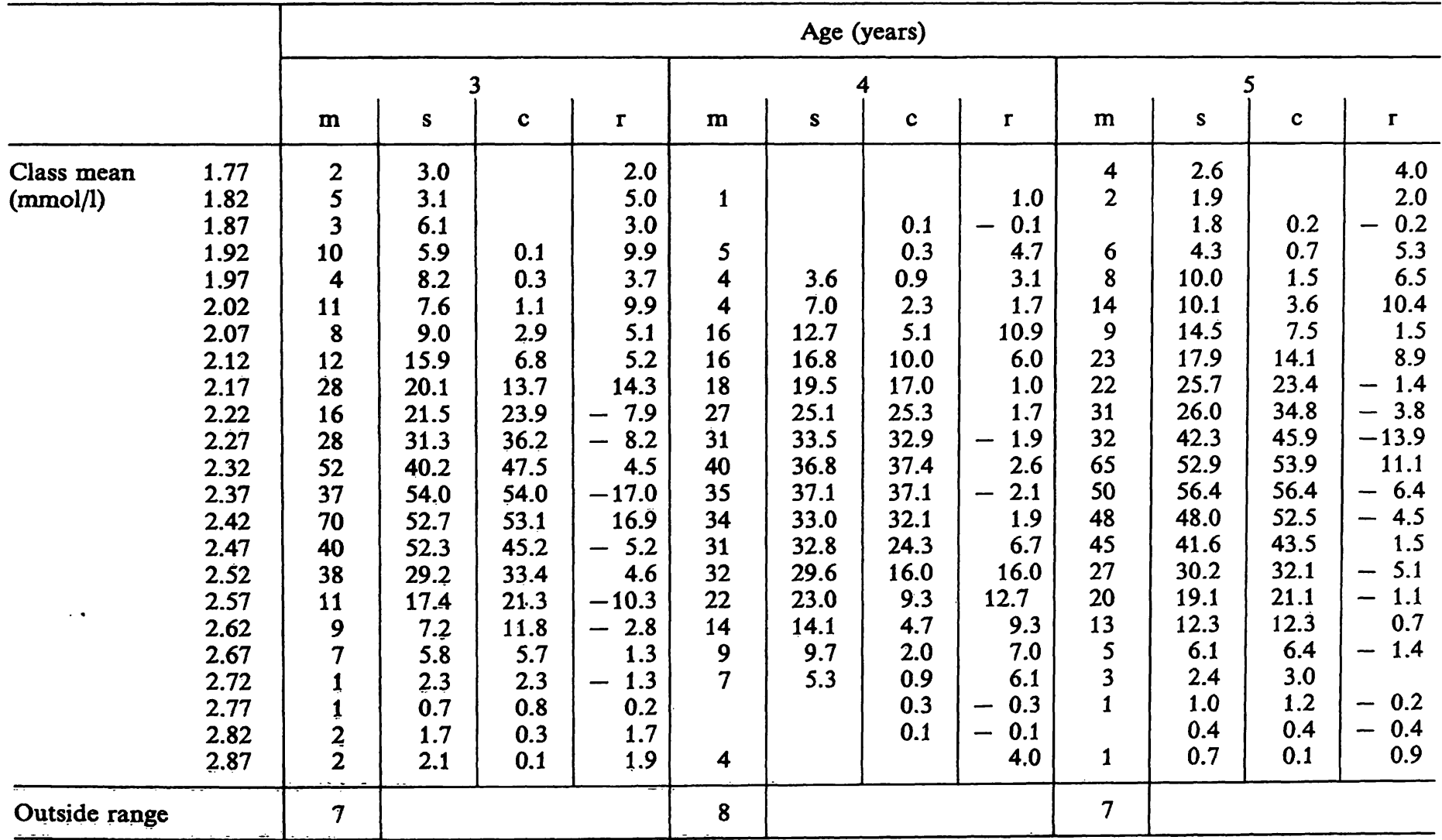

Distribution histograms for calcium

$m$ = measured data frequency

$s=$ smoothed data frequency

$c$ = calculated data frequency

$\mathbf{r}=$ residual data frequency (difference between measured and calculated data frequency) 
Calcium

\begin{tabular}{|c|c|c|c|c|c|c|c|c|c|c|c|c|c|}
\hline & \multicolumn{12}{|c|}{ Age (years) } \\
\hline & & \multicolumn{4}{|c|}{6} & \multicolumn{4}{|c|}{7} & \multicolumn{4}{|c|}{8} \\
\hline $\begin{array}{l}\text { Class mean } \\
(\mathrm{mmol} / \mathrm{l})\end{array}$ & $\begin{array}{l}1.77 \\
1.82 \\
1.87 \\
1.92 \\
1.97 \\
2.02 \\
2.07 \\
2.12 \\
2.17 \\
2.22 \\
2.27 \\
2.32 \\
2.37 \\
2.42 \\
2.47 \\
2.52 \\
2.57 \\
2.62 \\
2.67 \\
2.72 \\
2.77 \\
2.82 \\
2.87\end{array}$ & $\begin{array}{r}2 \\
3 \\
2 \\
2 \\
2 \\
6 \\
3 \\
14 \\
18 \\
26 \\
22 \\
33 \\
47 \\
43 \\
38 \\
24 \\
22 \\
9 \\
5 \\
3 \\
4\end{array}$ & $\begin{array}{r}2.2 \\
1.9 \\
1.4 \\
0.9 \\
3.4 \\
3.3 \\
6.6 \\
11.3 \\
20.3 \\
22.3 \\
25.3 \\
33.8 \\
43.7 \\
45.1 \\
35.5 \\
27.8 \\
18.3 \\
11.3 \\
4.3 \\
3.8 \\
2.6 \\
1.1 \\
0.1\end{array}$ & $\begin{array}{r}0.1 \\
0.4 \\
1.2 \\
3.2 \\
7.5 \\
14.8 \\
25.0 \\
35.8 \\
43.6 \\
45.1 \\
39.7 \\
29.6 \\
18.8 \\
10.2 \\
4.7 \\
1.8 \\
0.6 \\
0.2\end{array}$ & $\begin{array}{r}2.0 \\
3.0 \\
\\
2.0 \\
1.9 \\
5.6 \\
1.8 \\
10.8 \\
10.5 \\
11.2 \\
-3.0 \\
-2.8 \\
3.4 \\
-2.1 \\
-1.7 \\
-5.6 \\
3.2 \\
-1.2 \\
0.3 \\
1.2 \\
3.4 \\
-0.2\end{array}$ & $\begin{array}{r}1 \\
2 \\
5 \\
3 \\
5 \\
6 \\
14 \\
10 \\
12 \\
19 \\
31 \\
39 \\
47 \\
53 \\
32 \\
23 \\
9 \\
8 \\
\\
5 \\
3\end{array}$ & $\begin{array}{r}3.6 \\
4.2 \\
3.9 \\
8.3 \\
10.8 \\
11.6 \\
11.9 \\
20.0 \\
29.9 \\
39.5 \\
49.0 \\
47.5 \\
36.8 \\
20.0 \\
12.2 \\
4.6 \\
3.5 \\
2.8\end{array}$ & $\begin{array}{r}0.2 \\
0.6 \\
1.8 \\
4.8 \\
10.6 \\
20.0 \\
31.9 \\
42.9 \\
49.0 \\
47.3 \\
38.7 \\
26.8 \\
15.7 \\
7.8 \\
3.3 \\
1.2 \\
0.3 \\
0.1\end{array}$ & $\begin{array}{r}1.0 \\
2.0 \\
5.0 \\
3.0 \\
4.8 \\
5.4 \\
12.2 \\
5.2 \\
1.4 \\
-1.0 \\
-0.9 \\
-3.9 \\
-2.0 \\
5.7 \\
-6.7 \\
-3.8 \\
-6.7 \\
0.2 \\
-3.3 \\
3.8 \\
2.7 \\
-0.1\end{array}$ & $\begin{array}{r}1 \\
2 \\
2 \\
5 \\
9 \\
15 \\
16 \\
19 \\
23 \\
43 \\
42 \\
55 \\
43 \\
29 \\
24 \\
7 \\
4 \\
2 \\
\end{array}$ & $\begin{array}{r}2.5 \\
4.7 \\
9.7 \\
13.8 \\
16 . \overline{7} \\
17.6 \\
27.5 \\
36.8 \\
48.3 \\
49.7 \\
44.0 \\
31.7 \\
20.0 \\
10.3 \\
3.0 \\
1.7\end{array}$ & $\begin{array}{r}0.2 \\
0.7 \\
2.2 \\
5.9 \\
13.0 \\
24.0 \\
36.8 \\
46.9 \\
49.7 \\
43.8 \\
32.1 \\
19.6 \\
9.9 \\
4.2 \\
1.5 \\
0.4 \\
0.1\end{array}$ & $\begin{array}{r}1.0 \\
2.0 \\
2.0 \\
4.8 \\
8.3 \\
12.8 \\
10.1 \\
6.0 \\
-1.0 \\
6.2 \\
-4.9 \\
5.3 \\
-0.8 \\
-3.1 \\
4.4 \\
-2.9 \\
-0.2 \\
0.5 \\
-0.4 \\
0.9\end{array}$ \\
\hline Outside range & & 4 & & & & 1 & & & & 2 & & & \\
\hline
\end{tabular}

Calcium

\begin{tabular}{|c|c|c|c|c|c|c|c|c|c|c|c|c|c|}
\hline & & \multicolumn{12}{|c|}{ Age (years) } \\
\hline & & \multicolumn{4}{|c|}{9} & \multicolumn{4}{|c|}{10} & \multicolumn{4}{|c|}{11} \\
\hline & & $\mathrm{m}$ & $s$ & c & $\mathbf{r}$ & $\mathrm{m}$ & $\mathbf{s}$ & c & $r$ & $\mathbf{m}$ & $\mathbf{s}$ & c & $\mathbf{r}$ \\
\hline $\begin{array}{l}\text { Class mean } \\
(\mathrm{mmol} / \mathrm{l})\end{array}$ & $\begin{array}{l}1.77 \\
1.82 \\
1.87 \\
1.92 \\
1.97 \\
2.02 \\
2.07 \\
2.12 \\
2.17 \\
2.22 \\
2.27 \\
2.32 \\
2.37 \\
2.42 \\
2.47 \\
2.52 \\
2.57 \\
2.62 \\
2.67 \\
2.72 \\
2.77 \\
2.82 \\
2.87\end{array}$ & $\begin{array}{r}1 \\
2 \\
1 \\
5 \\
5 \\
17 \\
9 \\
12 \\
14 \\
27 \\
29 \\
48 \\
28 \\
24 \\
24 \\
16 \\
5 \\
2 \\
1 \\
1 \\
\\
1\end{array}$ & $\begin{array}{r}2.4 \\
2.9 \\
9.1 \\
11.6 \\
12.7 \\
9.9 \\
16.9 \\
22.7 \\
36.2 \\
38.5 \\
33.7 \\
24.0 \\
22.5 \\
15.5 \\
6.4 \\
1.6 \\
1.1\end{array}$ & $\begin{array}{r}0.1 \\
0.3 \\
1.0 \\
3.1 \\
7.7 \\
15.7 \\
26.1 \\
35.1 \\
38.5 \\
34.3 \\
24.8 \\
14.6 \\
7.0 \\
2.7 \\
0.9 \\
0.2\end{array}$ & $\begin{array}{r}1.0 \\
2.0 \\
1.0 \\
4.9 \\
4.7 \\
16.0 \\
5.9 \\
4.3 \\
-1.7 \\
0.9 \\
-6.1 \\
9.5 \\
-6.3 \\
-0.8 \\
9.4 \\
9.0 \\
2.3 \\
1.1 \\
0.8 \\
1.0 \\
1.0\end{array}$ & $\begin{array}{r}1 \\
2 \\
1 \\
2 \\
3 \\
4 \\
1 \\
12 \\
12 \\
22 \\
18 \\
29 \\
25 \\
46 \\
29 \\
23 \\
11 \\
5 \\
1 \\
2 \\
1\end{array}$ & $\begin{array}{r}1.4 \\
1.5 \\
1.8 \\
3.3 \\
2.1 \\
4.7 \\
8.1 \\
15.9 \\
17.4 \\
23.1 \\
23.0 \\
33.8 \\
36.4 \\
34.6 \\
20.5 \\
12.4 \\
4.4 \\
1.8 \\
1.2\end{array}$ & $\begin{array}{r}0.2 \\
0.7 \\
2.3 \\
6.1 \\
13.0 \\
22.6 \\
31.9 \\
36.4 \\
33.7 \\
25.3 \\
15.4 \\
7.6 \\
3.1 \\
1.0 \\
0.3\end{array}$ & $\begin{array}{r}1.0 \\
2.0 \\
1.0 \\
2.0 \\
3.0 \\
4.0 \\
0.8 \\
11.3 \\
9.7 \\
15.9 \\
5.0 \\
6.4 \\
-6.9 \\
9.6 \\
-4.7 \\
-2.3 \\
-4.4 \\
-2.6 \\
-2.1 \\
1.0 \\
0.7\end{array}$ & $\begin{array}{r}1 \\
1 \\
5 \\
4 \\
6 \\
12 \\
10 \\
15 \\
16 \\
22 \\
40 \\
43 \\
38 \\
32 \\
24 \\
5 \\
6 \\
2 \\
2 \\
1 \\
4\end{array}$ & $\begin{array}{r}3.2 \\
4.7 \\
7.1 \\
9.7 \\
12.2 \\
13.3 \\
16.2 \\
24.9 \\
37.1 \\
43.0 \\
38.7 \\
32.7 \\
20.6 \\
9.8 \\
3.0 \\
3.2 \\
1.5\end{array}$ & $\begin{array}{r}0.1 \\
0.4 \\
1.1 \\
2.8 \\
6.4 \\
12.4 \\
21.1 \\
30.9 \\
39.2 \\
43.0 \\
40.8 \\
33.5 \\
23.8 \\
14.7 \\
7.8 \\
3.6 \\
1.4 \\
0.5 \\
0.1\end{array}$ & $\begin{array}{r}1.0 \\
1.0 \\
4.9 \\
3.6 \\
4.9 \\
9.2 \\
3.6 \\
2.6 \\
-5.1 \\
-8.9 \\
0.8 \\
0.0 \\
-2.8 \\
-1.5 \\
0.2 \\
0.3 \\
-1.8 \\
-1.6 \\
0.6 \\
0.5 \\
-0.1 \\
4.0\end{array}$ \\
\hline Outside range & & 0 & & & & 1 & & & & 7 & & & \\
\hline
\end{tabular}

Distribution histograms for calcium (continued)

$\mathrm{m}=$ measured data frequency

$s$ = smoothed data frequency

c = calculated data frequency

$\mathbf{r}=$ residual data frequency (difference between measured and calculated data frequency) 
Colcium

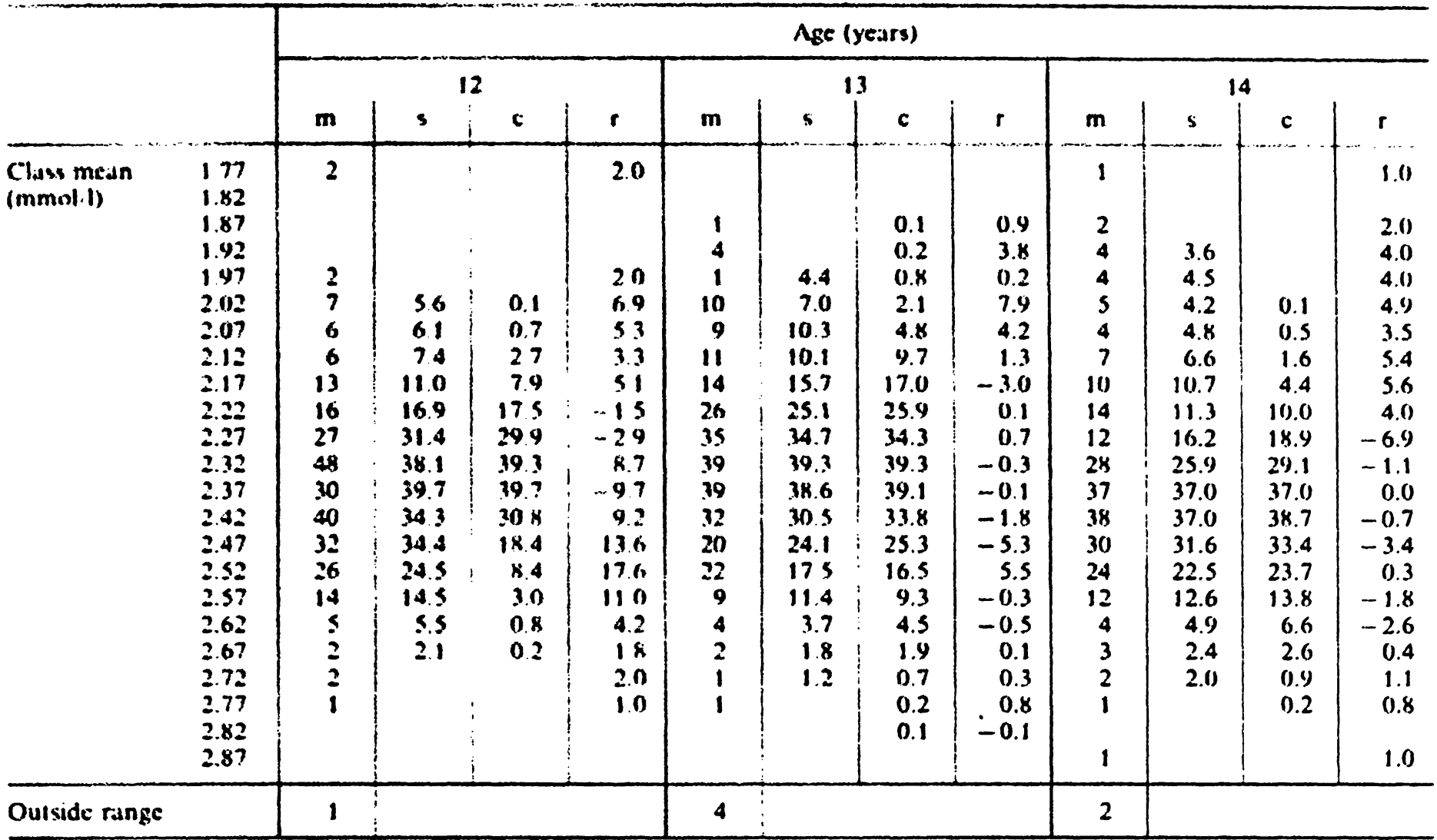

Calcium

\begin{tabular}{|c|c|c|c|c|c|c|c|c|c|c|c|c|c|}
\hline & & \multicolumn{12}{|c|}{ Age (years) } \\
\hline & & \multicolumn{4}{|c|}{15} & \multicolumn{4}{|c|}{16} & \multicolumn{4}{|c|}{$17-20$} \\
\hline & & $m$ & $\mathbf{s}$ & c & $\mathbf{r}$ & $\mathbf{m}$ & $\mathbf{s}$ & c & $r$ & $\mathbf{m}$ & $\mathbf{s}$ & c & $\mathbf{r}$ \\
\hline $\begin{array}{l}\text { Class mean } \\
(\mathrm{mmol} / 1)\end{array}$ & $\begin{array}{l}1.77 \\
1.82 \\
1.87 \\
1.92 \\
1.97 \\
2.02 \\
2.07 \\
2.12 \\
2.17 \\
2.22 \\
2.27 \\
2.32 \\
2.37 \\
2.42 \\
2.47 \\
2.52 \\
2.57 \\
2.62 \\
2.67 \\
2.72 \\
2.77 \\
2.82 \\
2.87\end{array}$ & $\begin{array}{r}2 \\
1 \\
6 \\
5 \\
16 \\
15 \\
21 \\
22 \\
20 \\
23 \\
20 \\
35 \\
40 \\
28 \\
23 \\
11 \\
8 \\
3 \\
2 \\
2\end{array}$ & $\begin{array}{r}3.4 \\
8.6 \\
12.3 \\
17.7 \\
19.8 \\
21.5 \\
21.6 \\
20.0 \\
24.5 \\
33.2 \\
37.3 \\
31.2 \\
20.4 \\
13.3 \\
6.5 \\
3.8 \\
2.0\end{array}$ & $\begin{array}{r}0.1 \\
0.6 \\
2.1 \\
6.1 \\
13.9 \\
24.7 \\
34.3 \\
37.3 \\
31.8 \\
21.3 \\
11.1 \\
4.6 \\
1.5 \\
0.4 \\
0.1\end{array}$ & $\begin{array}{r}2.0 \\
1.0 \\
6.0 \\
5.0 \\
16.0 \\
14.9 \\
20.4 \\
19.9 \\
13.9 \\
9.1 \\
-4.7 \\
0.7 \\
2.7 \\
-3.8 \\
1.7 \\
-0.1 \\
3.4 \\
1.5 \\
1.6 \\
1.9\end{array}$ & $\begin{array}{r}3 \\
2 \\
5 \\
7 \\
7 \\
9 \\
9 \\
8 \\
23 \\
31 \\
37 \\
29 \\
28 \\
19 \\
18 \\
14 \\
10 \\
3 \\
\\
3 \\
1\end{array}$ & $\begin{array}{r}4.7 \\
6.6 \\
7.7 \\
8.6 \\
7.6 \\
11.4 \\
20.6 \\
32.5 \\
34.2 \\
32.1 \\
25.3 \\
21.3 \\
16.8 \\
14.5 \\
9.1 \\
3.4 \\
1.1 \\
1.6\end{array}$ & $\begin{array}{r}0.2 \\
0.6 \\
2.0 \\
5.4 \\
11.8 \\
20.7 \\
29.6 \\
34.2 \\
32.0 \\
24.2 \\
14.8 \\
7.4 \\
3.0 \\
1.0 \\
0.3 \\
0.1\end{array}$ & $\begin{array}{r}3.0 \\
2.0 \\
5.0 \\
6.8 \\
6.4 \\
7.0 \\
3.6 \\
-3.8 \\
2.3 \\
2.4 \\
2.8 \\
-3.0 \\
3.8 \\
4.2 \\
10.6 \\
11.0 \\
9.0 \\
2.7 \\
-0.1 \\
3.0 \\
1.0\end{array}$ & $\begin{array}{r}3 \\
1 \\
3 \\
2 \\
5 \\
11 \\
14 \\
17 \\
27 \\
26 \\
29 \\
26 \\
19 \\
18 \\
11 \\
6 \\
3 \\
1 \\
1 \\
1 \\
1\end{array}$ & $\begin{array}{r}1.7 \\
2.8 \\
5.4 \\
10.2 \\
13.7 \\
19.1 \\
24.2 \\
28.1 \\
28.0 \\
25.3 \\
20.9 \\
16.3 \\
11.7 \\
6.1 \\
2.9\end{array}$ & $\begin{array}{r}0.1 \\
0.2 \\
0.6 \\
1.3 \\
2.8 \\
5.3 \\
9.1 \\
14.1 \\
19.7 \\
24.8 \\
28.1 \\
28.8 \\
26.5 \\
22.0 \\
16.4 \\
11.1 \\
6.7 \\
3.7 \\
1.8 \\
0.8 \\
0.3 \\
0.1\end{array}$ & $\begin{array}{r}3.0 \\
-0.1 \\
0.8 \\
-0.6 \\
1.7 \\
-0.8 \\
-0.3 \\
1.8 \\
-0.1 \\
-2.7 \\
2.2 \\
-2.1 \\
0.2 \\
-0.5 \\
-3.0 \\
1.6 \\
-0.1 \\
-0.7 \\
-0.7 \\
-0.8 \\
-0.8 \\
0.7 \\
0.9\end{array}$ \\
\hline Outside range & & 8 & & & & 1 & & & & 0 & & & \\
\hline
\end{tabular}

Distribution histograms for calcium (continued)

$m=$ measured data frequency

$s$ = smoothed data frequency

c = calculated data frequency

$r$ = residual data frequency (difference between measured and calculated data frequency) 
Magnesium

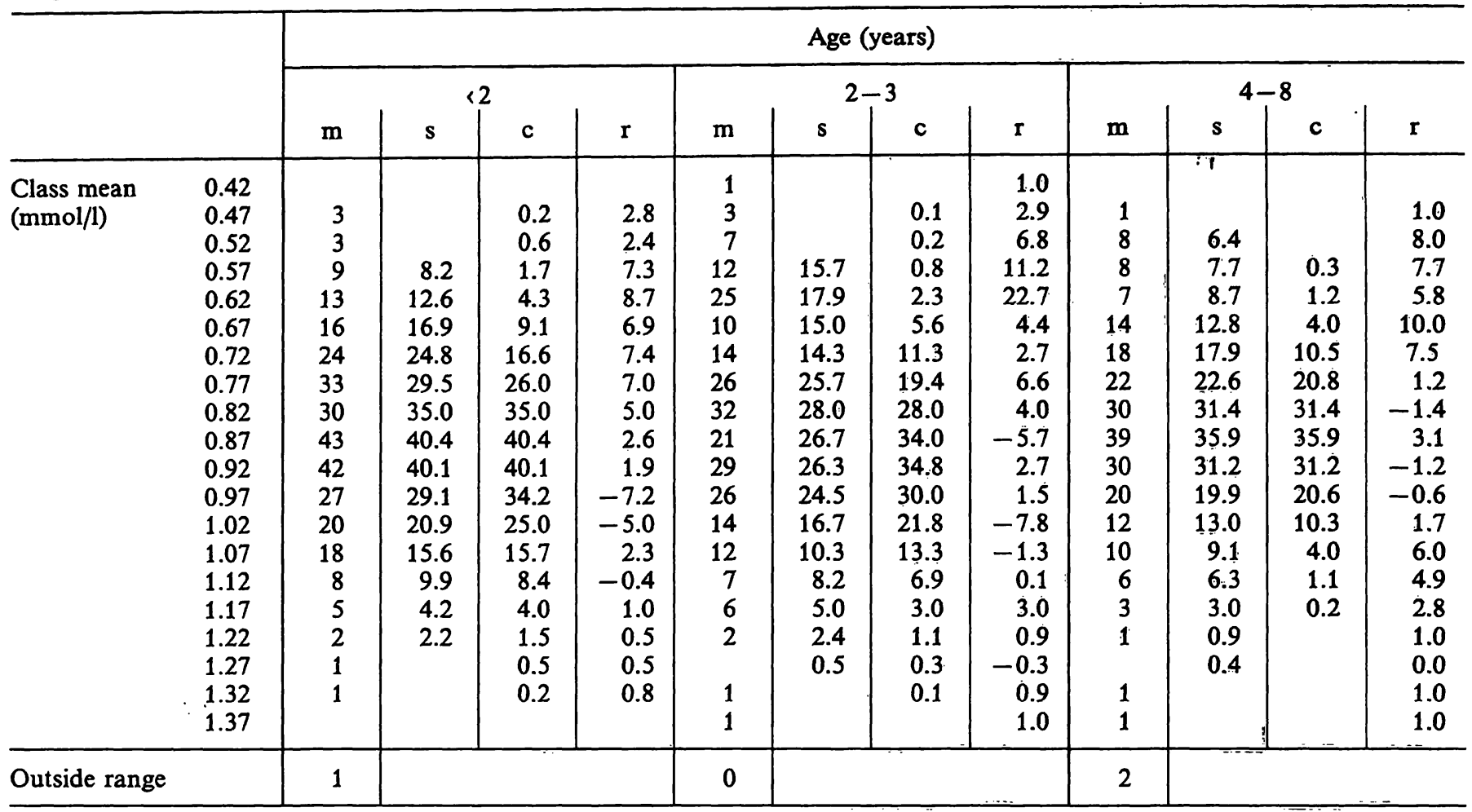

Magnesium

\begin{tabular}{|c|c|c|c|c|c|c|c|c|c|}
\hline & & \multicolumn{8}{|c|}{ Age (years) } \\
\hline & & \multicolumn{4}{|c|}{$9-13$} & \multicolumn{4}{|c|}{$14-20$} \\
\hline & & m & $\mathbf{s}$ & c & $\mathbf{r}$ & $\mathbf{m}$ & $s$ & c & $\mathbf{r}$ \\
\hline $\begin{array}{l}\text { Class mean } \\
(\mathrm{mmol} / \mathrm{l})\end{array}$ & $\begin{array}{l}0.42 \\
0.47 \\
0.52 \\
0.57 \\
0.62 \\
0.67 \\
0.72 \\
0.77 \\
0.82 \\
0.87 \\
0.92 \\
0.97 \\
1.02 \\
1.07 \\
1.12 \\
1.17 \\
1.22 \\
1.27 \\
1.32 \\
1.37\end{array}$ & $\begin{array}{r}8 \\
9 \\
4 \\
9 \\
10 \\
25 \\
32 \\
34 \\
34 \\
19 \\
16 \\
9 \\
7 \\
3 \\
\\
2 \\
\\
2\end{array}$ & $\begin{array}{r}6.6 \\
6.3 \\
13.4 \\
22.8 \\
32.0 \\
35.4 \\
30.6 \\
22.7 \\
13.9 \\
10.4 \\
6.1 \\
2.9 \\
1.2\end{array}$ & $\begin{array}{r}0.2 \\
0.7 \\
2.4 \\
6.4 \\
13.6 \\
23.2 \\
32.0 \\
35.4 \\
31.5 \\
22.6 \\
13.1 \\
6.1 \\
2.3 \\
0.7 \\
0.2\end{array}$ & $\begin{array}{r}7.8 \\
8.3 \\
1.6 \\
2.6 \\
-3.6 \\
1.8 \\
0.0 \\
-1.4 \\
2.5 \\
-3.6 \\
2.9 \\
2.9 \\
4.7 \\
2.3 \\
-0.2 \\
2.0 \\
0.0 \\
2.0\end{array}$ & $\begin{array}{r}1 \\
2 \\
2 \\
5 \\
18 \\
19 \\
24 \\
27 \\
25 \\
30 \\
29 \\
18 \\
7 \\
5 \\
4 \\
2 \\
3\end{array}$ & $\begin{array}{r}7.5 \\
14.7 \\
20.9 \\
23.7 \\
25.7 \\
27.1 \\
29.2 \\
27.8 \\
18.1 \\
8.5 \\
4.5 \\
3.5 \\
2.9\end{array}$ & $\begin{array}{r}0.2 \\
1.1 \\
3.5 \\
8.9 \\
17.3 \\
25.7 \\
29.2 \\
25.5 \\
17.0 \\
8.7 \\
3.4 \\
1.0 \\
0.2\end{array}$ & $\begin{array}{r}1.0 \\
2.0 \\
2.0 \\
4.8 \\
16.9 \\
15.5 \\
15.1 \\
9.7 \\
-0.7 \\
0.8 \\
3.5 \\
1.0 \\
-1.7 \\
1.6 \\
3.0 \\
1.8 \\
3.0 \\
0.0 \\
0.0 \\
1.0\end{array}$ \\
\hline Outside range & & 2 & & & & 3 & & & \\
\hline
\end{tabular}

Distribution histograms for magnesium

$\mathrm{m}=$ measured data frequency

$\mathbf{s}=$ smoothed data frequency

c = calculated data frequency

$\mathbf{r}=$ residual data frequency (difference between measured and calculated data frequency) 
Inotg.snic phospharuv

\begin{tabular}{|c|c|c|c|c|c|c|c|c|c|c|c|c|c|}
\hline & & \multicolumn{12}{|c|}{ Age (yearss) } \\
\hline & & m & 5 & C & $r$ & $\mathbf{m}$ & $s$ & C & $r$ & $m$ & s & c & $\mathbf{r}$ \\
\hline $\begin{array}{l}\text { ('lass mean } \\
\text { (mmol!l) }\end{array}$ & $\begin{array}{l}0.45 \\
0.55 \\
065 \\
075 \\
0.85 \\
095 \\
1.05 \\
115 \\
125 \\
1.35 \\
1.45 \\
1.55 \\
1.65 \\
1.75 \\
1.85 \\
1.95 \\
205 \\
2.15 \\
2.25 \\
2.35 \\
2.45 \\
2.55\end{array}$ & $\begin{array}{c}1 \\
2 \\
1 \\
2 \\
3 \\
2 \\
5 \\
13 \\
12 \\
13 \\
14 \\
15 \\
26 \\
42 \\
44 \\
40 \\
23 \\
14 \\
9 \\
6 \\
3\end{array}$ & $\begin{array}{r}1.1 \\
1.6 \\
20 \\
2.3 \\
2.4 \\
6.3 \\
10.9 \\
13.1 \\
12.8 \\
13.1 \\
16.3 \\
27.2 \\
40.5 \\
41.9 \\
32.7 \\
217 \\
14.7 \\
4.0 \\
5.8 \\
2.7\end{array}$ & $\begin{array}{r}0.1 \\
0.2 \\
0.7 \\
2.1 \\
5.1 \\
10.5 \\
18.7 \\
28.5 \\
37.3 \\
41.9 \\
40.4 \\
33.4 \\
23.7 \\
14.4 \\
7.6 \\
3.4\end{array}$ & $\begin{array}{r}1.0 \\
0.0 \\
2.0 \\
1.0 \\
2.0 \\
3.0 \\
1.9 \\
4.8 \\
12.3 \\
9.9 \\
7.9 \\
3.5 \\
-3.7 \\
-2.5 \\
4.7 \\
2.1 \\
-10.4 \\
-10.4 \\
-\quad 9.7 \\
-5.4 \\
-1.6 \\
-10.4\end{array}$ & $\begin{array}{r}1 \\
3 \\
6 \\
3 \\
18 \\
12 \\
6 \\
20 \\
29 \\
22 \\
49 \\
62 \\
70 \\
51 \\
54 \\
17 \\
16 \\
1 \\
4 \\
1\end{array}$ & $\begin{array}{r}32 \\
3.4 \\
\times 4 \\
12.9 \\
121 \\
9.9 \\
18.8 \\
23.8 \\
30.4 \\
44.1 \\
647 \\
63.9 \\
611.5 \\
42.2 \\
27.2 \\
11.4 \\
9.2 \\
3.6\end{array}$ & $\begin{array}{r}0.1 \\
0.4 \\
17 \\
5.4 \\
13.7 \\
28.4 \\
47.6 \\
64.7 \\
71.1 \\
63.3 \\
45.7 \\
27.0 \\
12.6 \\
4.8 \\
1.5 \\
0.4\end{array}$ & $\begin{array}{r}1.0 \\
00 \\
3.0 \\
6.0 \\
3.0 \\
17.9 \\
11.6 \\
4.3 \\
14.6 \\
15.3 \\
-\quad 6.4 \\
1.4 \\
-\quad 2.7 \\
-1.1 \\
-12.3 \\
8.3 \\
-10.0 \\
3.4 \\
3.2 \\
2.5 \\
0.6\end{array}$ & $\begin{array}{r}1 \\
1 \\
1 \\
3 \\
1 \\
11 \\
11 \\
13 \\
14 \\
23 \\
36 \\
47 \\
51 \\
40 \\
29 \\
18 \\
7 \\
3 \\
1 \\
1 \\
1\end{array}$ & $\begin{array}{r}1.7 \\
1.1 \\
4.3 \\
8.1 \\
12.3 \\
12.0 \\
15.1 \\
23.2 \\
35.9 \\
47.3 \\
49.0 \\
41.3 \\
29.0 \\
17.4 \\
8.0 \\
2.6 \\
1.2 \\
0.9\end{array}$ & $\begin{array}{r}0.1 \\
0.5 \\
1.8 \\
5.2 \\
12.1 \\
23.3 \\
36.5 \\
46.8 \\
49.0 \\
42.0 \\
29.3 \\
16.8 \\
7.8 \\
3.0 \\
0.9 \\
0.2\end{array}$ & $\begin{array}{r}1.0 \\
1.0 \\
1.0 \\
3.11 \\
2.9 \\
10.5 \\
0.2 \\
7.8 \\
1.9 \\
0.3 \\
-0.5 \\
0.2 \\
2.0 \\
.2 .0 \\
-0.3 \\
1.2 \\
-0.8 \\
0.0 \\
0.1 \\
0.8 \\
1.0\end{array}$ \\
\hline Outside range & & 5 & & & & 0 & & & & 2 & & & \\
\hline
\end{tabular}

Inorganic phosphorus

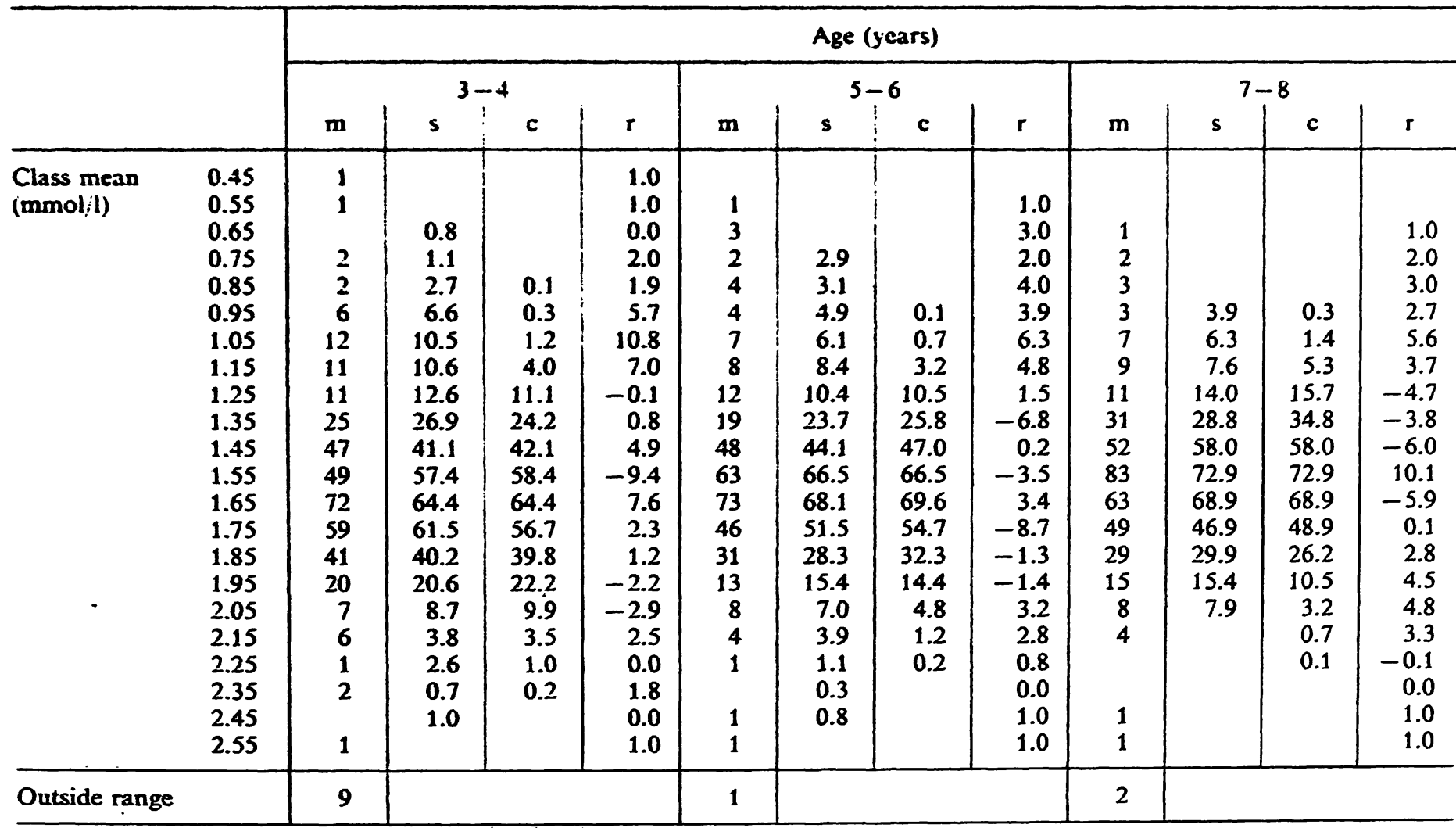

Distribution histograms for inorganic phosphorus

$\mathrm{m}=$ measured data frequency

$s$ = smoothed data frequency

$c$ = calculated data frequency

$r$ = residual data frequency (difference between measured and calculated data frequency) 
Inorganic phosphorus

\begin{tabular}{|c|c|c|c|c|c|c|c|c|c|}
\hline & & \multicolumn{8}{|c|}{ Age (years) } \\
\hline & & \multicolumn{4}{|c|}{$9-10$} & \multicolumn{4}{|c|}{$11-12$} \\
\hline & & $\mathbf{m}$ & $\mathbf{s}$ & c & $\mathbf{r}$ & $\mathbf{m}$ & $\mathbf{s}$ & c & $\mathbf{r}$ \\
\hline $\begin{array}{l}\text { Class mean } \\
(\mathrm{mmol} / \mathrm{l})\end{array}$ & $\begin{array}{l}0.45 \\
0.55 \\
0.65 \\
0.75 \\
0.85 \\
0.95 \\
1.05 \\
1.15 \\
1.25 \\
1.35 \\
1.45 \\
1.55 \\
1.65 \\
1.75 \\
1.85 \\
1.95 \\
2.05 \\
2.15 \\
2.25 \\
2.35 \\
2.45 \\
2.55\end{array}$ & $\begin{array}{r}1 \\
1 \\
1 \\
3 \\
2 \\
5 \\
9 \\
14 \\
28 \\
50 \\
69 \\
56 \\
34 \\
18 \\
6 \\
3 \\
\\
3 \\
1 \\
1\end{array}$ & $\begin{array}{r}2.0 \\
2.9 \\
4.7 \\
8.3 \\
14.8 \\
28.9 \\
51.5 \\
64.5 \\
56.7 \\
35.5 \\
17.4 \\
7.2 \\
1.7 \\
1.5 \\
1.5\end{array}$ & $\begin{array}{r}0.1 \\
0.8 \\
3.7 \\
12.4 \\
29.9 \\
51.8 \\
64.5 \\
57.8 \\
37.3 \\
17.3 \\
5.8 \\
1.4 \\
0.2\end{array}$ & $\begin{array}{r}1.0 \\
1.0 \\
1.0 \\
3.0 \\
1.9 \\
4.2 \\
5.3 \\
1.6 \\
-1.9 \\
-1.8 \\
4.5 \\
-1.8 \\
-3.3 \\
0.7 \\
0.2 \\
1.6 \\
-0.2 \\
3.0 \\
1.0 \\
1.0\end{array}$ & $\begin{array}{r}2 \\
1 \\
5 \\
5 \\
5 \\
3 \\
14 \\
9 \\
38 \\
60 \\
55 \\
54 \\
30 \\
7 \\
4 \\
2 \\
\\
1 \\
\\
1 \\
1\end{array}$ & $\begin{array}{r}4.0 \\
6.8 \\
7.2 \\
16.8 \\
36.2 \\
55.6 \\
60.0 \\
49.6 \\
30.4 \\
10.3 \\
2.4 \\
1.7 \\
0.7 \\
0.3 \\
0.6\end{array}$ & $\begin{array}{r}0.2 \\
1.3 \\
5.5 \\
16.3 \\
35.0 \\
54.0 \\
60.0 \\
48.0 \\
27.7 \\
11.5 \\
3.4 \\
0.7 \\
0.1\end{array}$ & $\begin{array}{r}11 \\
2.0 \\
1.0 \\
0.0 \\
5.0 \\
4.8 \\
1.7 \\
8.5 \\
-7.3 \\
3.0 \\
6.0 \\
-5.0 \\
6.0 \\
2.3 \\
-4.5 \\
0.6 \\
1.3 \\
-0.1 \\
1.0 \\
0.0 \\
1.0 \\
1.0\end{array}$ \\
\hline Outside range & & 0 & & & & 3 & & & \\
\hline
\end{tabular}

Inorganic phosphorus

\begin{tabular}{|c|c|c|c|c|c|c|c|c|c|}
\hline & & \multicolumn{8}{|c|}{ Age (years) } \\
\hline & & \multicolumn{4}{|c|}{$13-14$} & \multicolumn{4}{|c|}{$15-20$} \\
\hline & & $\mathbf{m}$ & $\mathbf{s}$ & c & $\mathbf{r}$ & $\mathbf{m}$ & $\mathbf{s}$ & c & r \\
\hline $\begin{array}{l}\text { Class mean } \\
(\mathrm{mmol} / \mathrm{l})\end{array}$ & $\begin{array}{l}0.45 \\
0.55 \\
0.65 \\
0.75 \\
0.85 \\
0.95 \\
1.05 \\
1.15 \\
1.25 \\
1.35 \\
1.45 \\
1.55 \\
1.65 \\
1.75 \\
1.85 \\
1.95 \\
2.05 \\
2.15 \\
2.25 \\
2.35 \\
2.45 \\
2.55\end{array}$ & $\begin{array}{r}3 \\
5 \\
11 \\
32 \\
43 \\
35 \\
42 \\
41 \\
24 \\
16 \\
9 \\
3 \\
1 \\
4 \\
1 \\
1\end{array}$ & $\begin{array}{r}14.1 \\
30.6 \\
39.3 \\
39.9 \\
40.7 \\
38.2 \\
26.8 \\
15.3 \\
8.7 \\
3.2 \\
2.0 \\
2.3\end{array}$ & $\begin{array}{r}0.1 \\
0.7 \\
2.7 \\
7.8 \\
17.5 \\
30.0 \\
39.5 \\
39.9 \\
30.9 \\
18.3 \\
8.3 \\
3.0 \\
0.8 \\
0.2\end{array}$ & $\begin{array}{r}-0.1 \\
-0.7 \\
0.3 \\
-2.8 \\
-6.5 \\
2.0 \\
3.5 \\
-4.9 \\
11.1 \\
22.7 \\
15.7 \\
13.0 \\
8.2 \\
2.8 \\
1.0 \\
4.0 \\
1.0 \\
1.0\end{array}$ & $\begin{array}{r}1 \\
2 \\
2 \\
7 \\
7 \\
16 \\
25 \\
33 \\
58 \\
55 \\
33 \\
37 \\
22 \\
5 \\
5 \\
5 \\
2 \\
2 \\
\\
1\end{array}$ & $\begin{array}{r}7.3 \\
15.7 \\
23.4 \\
38.4 \\
53.4 \\
51.9 \\
40.7 \\
31.7 \\
21.8 \\
8.1 \\
3.8 \\
4.2 \\
2.9 \\
1.2 \\
0.9\end{array}$ & $\begin{array}{r}0.2 \\
0.9 \\
3.6 \\
10.5 \\
23.6 \\
40.5 \\
53.4 \\
53.9 \\
41.7 \\
24.7 \\
11.2 \\
4.0 \\
1.0 \\
0.2\end{array}$ & $\begin{array}{r}1.0 \\
1.8 \\
1.1 \\
3.4 \\
5.5 \\
1.4 \\
-7.5 \\
4.6 \\
1.1 \\
-8.7 \\
12.3 \\
9.8 \\
1.0 \\
4.0 \\
4.8 \\
2.0 \\
2.0 \\
0.0 \\
1.0\end{array}$ \\
\hline Outside range & & 2 & & & & 4 & & & . \\
\hline
\end{tabular}

Distribution histograms for inorganic phosphorus (continued)

$\mathrm{m}=$ measured data frequency

$s=$ smoothed data frequency

c = calculated data frequency

$r$ = residual data frequency (difference between measured and calculated data frequehcy) 